\title{
The knockout of miR-143 and -145 alters smooth muscle cell maintenance and vascular homeostasis in mice: correlates with human disease
}

\author{
L Elia ${ }^{1,2,6}$, M Quintavalle ${ }^{3,6}$, J Zhang ${ }^{1}$, R Contu ${ }^{2,4}$, L Cossu ${ }^{4}$, MVG Latronico ${ }^{4}$, KL Peterson ${ }^{1}$, C Indolfi ${ }^{5}$, D Catalucci ${ }^{2,4}$, J Chen ${ }^{1}$, \\ SA Courtneidge $\mathrm{e}^{\star, 3}$ and G Condorelli ${ }^{*, 1,2,4}$
}

Mechanisms controlling vascular smooth muscle cell (VSMC) plasticity and renewal still remain to be elucidated completely. A class of small RNAs called microRNAs (miRs) regulate gene expression at the post-transcriptional level. Here, we show a critical role of the miR-143/145 cluster in SMC differentiation and vascular pathogenesis, also through the generation of a mouse model of miR-143 and -145 knockout (KO). We determined that the expression of miR-143 and -145 is decreased in acute and chronic vascular stress (transverse aortic constriction and in aortas of the ApoE KO mouse). In human aortic aneurysms, the expression of miR-143 and -145 was significantly decreased compared with control aortas. In addition, overexpression of miR-143 and -145 decreased neointimal formation in a rat model of acute vascular injury. An in-depth analysis of the miR-143/145 KO mouse model showed that this miR cluster is expressed mostly in the SMC compartment, both during development and postnatally, in vessels and SMC-containing organs. Loss of miR-143 and miR-145 expression induces structural modifications of the aorta, because of an incomplete differentiation of VSMCs. In conclusion, our results show that the miR-143/145 gene cluster has a critical role during SMC differentiation and strongly suggest its involvement in the reversion of the VSMC differentiation phenotype that occurs during vascular disease.

Cell Death and Differentiation (2009) 16, 1590-1598; doi:10.1038/cdd.2009.153; published online 9 October 2009

In the postnatal organism, vascular smooth muscle cells (VSMCs) - unlike muscle cells of the skeletal and cardiac lineage - switch from a differentiated (contractile) to a dedifferentiated (synthetic) phenotype when subjected to appropriate environmental cues, allowing them to proliferate and migrate. ${ }^{1,2}$ Striking examples of SMC plasticity can be seen during vascular development, when SMCs have a key function in morphogenesis of blood vessels. During this phase, they exhibit high rates of proliferation and migration, and produce extracellular matrix (ECM) components that make up a major portion of the blood vessel wall, such as collagen, elastin, and proteoglycans. At the same time SMCs acquire contractile capabilities. Dysregulation of this extraordinary plasticity of VSMCs underlies the pathogenesis of human diseases such as atherosclerosis ${ }^{3}$ hampers vascular reconstruction procedures by the perpetuation of restenosis, ${ }^{3}$ and has an important function in the neovascularization of tumors and in age-related vascular decay. ${ }^{4}$

Various protein-based signaling cascades have been implicated in controlling VSMC differentiation, but the mechanisms that regulate the VSMC phenotype remain partially understood. ${ }^{5}$ Research has only recently started to focus on microRNAs (miRs) in the heart ${ }^{6}$ and vasculature. ${ }^{7}$ This class of post-transcriptional regulator comprises B22 nucleotide long RNAs that negatively regulate gene expression by binding to sites present in the $3^{\prime}$ untranslated region of targeted mRNAs. ${ }^{8}$ To date, over 700 different miRs have been found in humans (http://www.mirbase.org/). Bioinformatic analyses predict that each miR might regulate hundreds of targets, suggesting that miRs are likely to have roles in almost all biological processes. ${ }^{9}$

Dedifferentiated VSMCs in the adult are characterized by reduced expression of some SMC-related genes such as smooth muscle $\alpha$-actin, acquisition of increased rates of proliferation and motility, and the ability to synthesize collagens and matrix metalloproteases. 5,10

The present report documents a role for miR-143 and -145 genes in maintaining the differentiation status of VSMCs and controlling VSMC motility. Dysregulation of the miR-143 and -145 genes is suggested to underlie the inappropriate SMC plasticity observed with vascular disease, by promoting a phenotypic switch from a differentiated to an undifferentiated, proliferative and migratory state.

\section{Results}

miR-143/145 expression in the aorta. In a screen for miR expression in different tissues we found that miR-143 was

${ }^{1}$ Division of Cardiology, Department of Medicine, University of California San Diego, 9500 Gilman Drive, La Jolla, CA 92093-0613C, USA; ${ }^{2}$ Institute of Biomedical Technologies, Consiglio Nazionale delle Ricerche, via Fratelli Cervi 93, Milan 20138, Italy; ${ }^{3}$ Burnham Institute for Medical Research, 10901 North Torrey Pines Road, La Jolla, CA 92037, USA; ${ }^{4}$ I.R.C.C.S. MultiMedica Hospital, via Gaudenzio Fantoli 16/15, Milan 20138, Italy and ${ }^{5}$ University Magna Graecia, Catanzaro 88100 , Italy ${ }^{*}$ Corresponding authors. G Condorelli, Division of Cardiology, Department of Medicine, University of California San Diego, 9500 Gilman Drive, La Jolla, CA 92093-0613C, USA. Tel: + 1858822 5563; Fax: + 1858822 3027; E-mail: gcondorelli @ ucsd.edu or SA Courtneidge, Burnham Institute for Medical Research, 10901 North Torrey Pines Road, La Jolla, CA 92037, USA. Tel: +1 858646 3128; Fax: +1 85879 5526; E-mail: courtneidge @ burnham.org

${ }^{6}$ These authors contributed equally to this work.

Keywords: microRNA; vascular smooth muscle cells; cell migration

Abbreviations: VSMC, vascular smooth muscle cell; miRs, microRNAs; UTR, untranslated region

Received 31.8.09; revised 16.9.09; accepted 16.9.09; Edited by G Melino; published online 9.10.09 
a

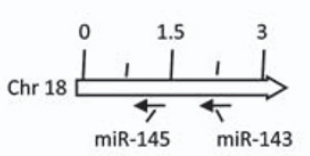

C
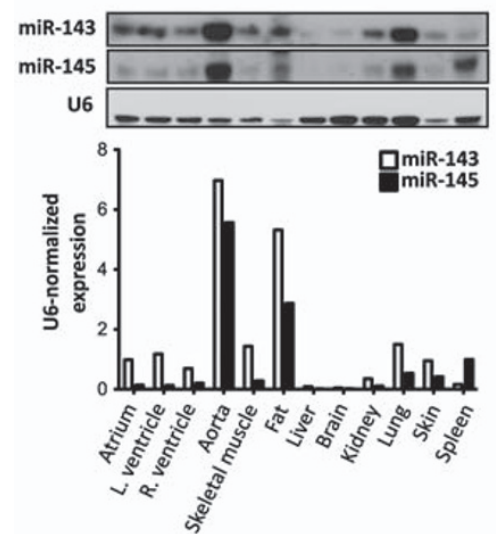

b

Mouse

Rat

Human

Rhesus

Chicken

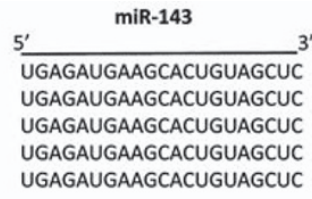

5

GUCCAGUUUUCCCAGGAAUCCCU GUCCAGUUUUCCCAGGAAUCCCU GUCCAGUUUUCCCAGGAAUCCCU GUCCAGUUUUCCCAGGAAUCCCU GUCCAGUUUUCCCAGGAAUCCCU

\section{d}

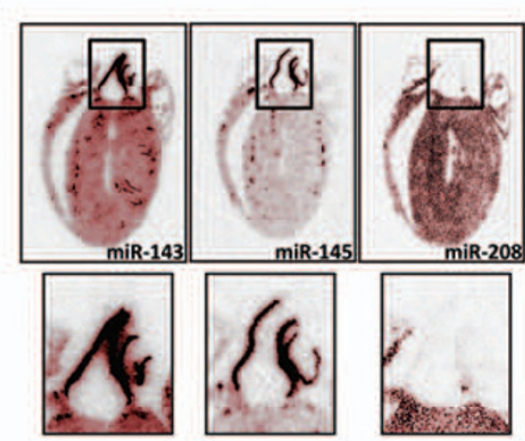

Figure 1 Expression of miR-143 and miR-145. (a) Localization of miR-143 and miR-145 in the genome of the mouse. (b) miR-143 and -145 sequence alignment in different species. (c) Representative northern blot of miR-143 and miR-145 in different tissues (top); band intensities were quantified using ImageJ software version 1.34 (http://rsb.info.nih.gov/ij/) and normalized to U6 (bottom). (d) Radioactive in situ hybridization for miR-143, -145 and -208 on adult mouse heart. Hearts were pseudo-colored in red to enhance the contrast using Adobe Photoshop

more expressed in the heart than in other organs (data not shown). We therefore decided to address the importance of miR-143 and its cluster partner, miR-145, in cardiovascular biology (Figure 1a and b). First, we analyzed the pattern of expression of miR-143 and miR-145 in various mouse tissues by northern blotting and found that miR-143 is present in lung, skeletal muscle, heart, skin and most abundant in aorta and fat, where miR-145 is also at its highest level (Figure 1c). Consistent with this, in situ hybridization of cross-sections of the adult mouse heart revealed a very strong signal for miR-143 and -145 in the walls of the aorta and coronary vessels (Figure 1d).

As many miRs become mis-expressed during disease, we decided to apply stress to the cardiovascular system to uncover whether miR-143 and miR-145 are involved in cardiovascular pathology. To this end, we analyzed the expression of miR-143 and miR-145 in wild-type (WT) mice subjected to transverse aortic constriction (TAC), a procedure used to generate stress on myocardial cells through pressure overload. ${ }^{11}$ After TAC, myocardial expression of miR-143 and miR-145, measured by quantitative real-time reverse polymerase chain reaction (qRT-PCR), did not change (data not shown); in contrast, expression of these miRs was dramatically reduced in the aorta upstream and downstream of the TAC site (Figure 2a).

We then determined miR-143 and miR-145 expression in a second model of vascular stress, the apolipoprotein $E$ (ApoE) knockout (KO) mouse, a mouse model of atherosclerosis, in which vascular damage is enhanced by a hypercholesterolaemic diet. ${ }^{12}$ qRT-PCR revealed that the aorta of ApoE KO mice had markedly decreased constitutive levels of miR-143 and miR-145, and that expression was further reduced (to less than $75 \%$ of WT levels) when fed on a high-fat diet (HFD) (Figure 2b). These data show that expression of miR-143 and miR-145 is regulated in adult mice by acute and chronic stress, suggesting a role for these miRs in the pathogenesis of vascular degeneration.

Next, we evaluated whether the expression of these miRs is changed in human disease. Biopsies were collected during surgical treatment of $(n=12)$ patients presenting with aortic aneurysm (ascending aortic diameter $>55 \mathrm{~mm}$ ) and subjected to qRT-PCR. As a control group, aortic biopsies were obtained from $(n=5)$ patients with normal ascending aortic diameter. We found that the diseased group had a significant decrease in expression of both miR-143 and -145 compared with the control group (Figure 2c). This result suggests that expression of the miR-143/145 gene could be probably downregulated in human vascular disease.

Analysis of a miR-143(145) KO mouse. To study the biology of miR-143 and miR-145, we generated a KO mouse model in which the exon specifying miR-143 was substituted by the lacZ reporter gene ${ }^{13}$ (Figure $3 a$ and $b$ ). This construct allows the visualization of cells expressing miR-143 and -145 through $\beta$-galactosidase staining. qRT-PCR demonstrated a negligible level of miR-143 in the aorta and other organs of homozygous KO mice (data not shown), which was confirmed by northern blotting (Figure $3 \mathrm{c}$ ). The expression of miR-145 was concomitantly affected by the loss of miR143 because both miR-143 and miR-145 originate from the same transcriptional unit and from the same primary miR. ${ }^{14}$ For this reason, we will refer to this mouse model as the miR143(145) KO. 

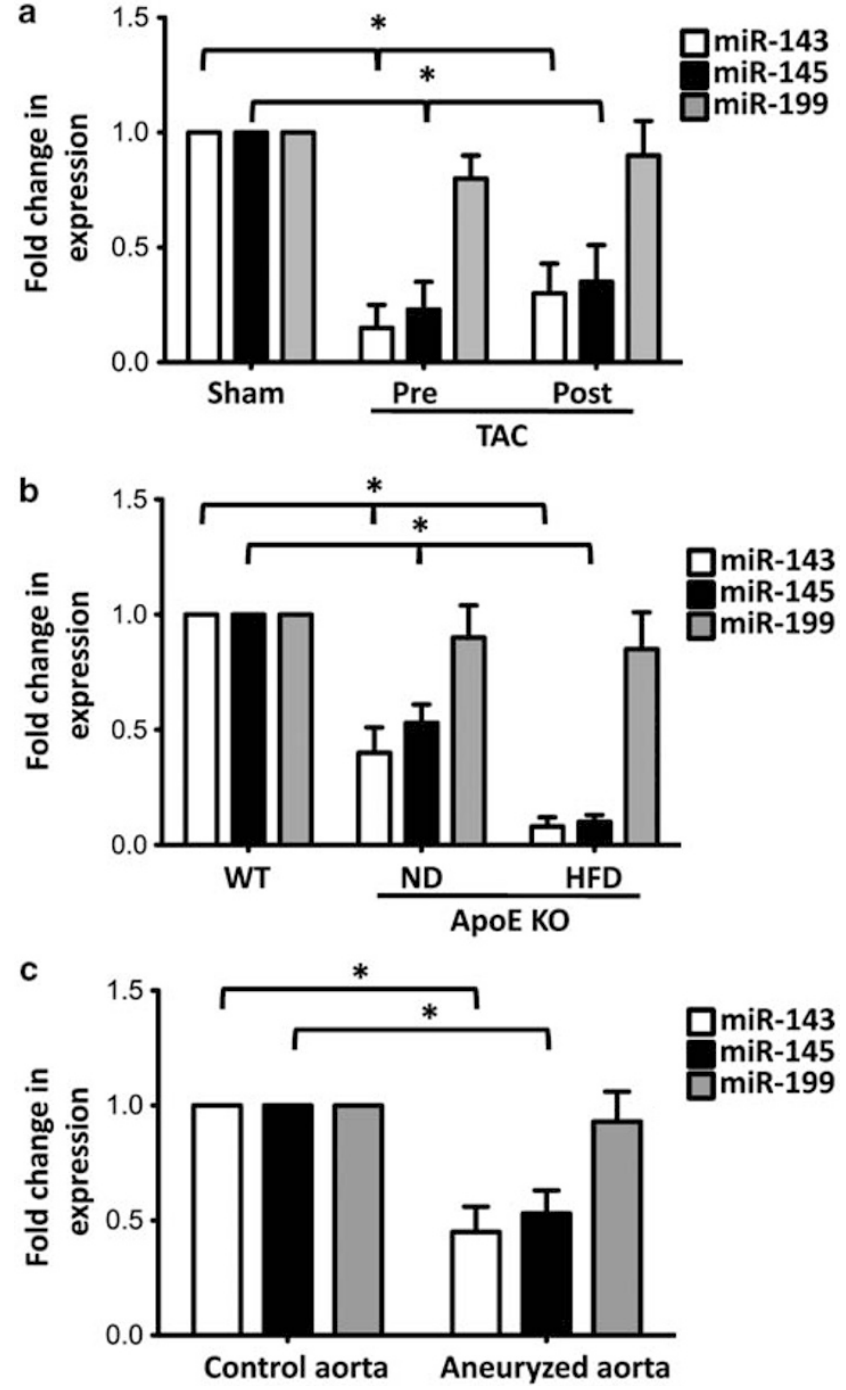

Figure 2 Expression of miR-143 and miR-145 in vascular diseases. (a) qRTPCR of samples of aorta from sham and pressure-overloaded mice generated by aortic constriction (TAC); Pre, portion of aorta proximal to the constriction; Post, portion of an aorta distal to the constriction; Sno22 RNA was used as internal control; All measurements were calculated as percent of control (Sham) and error bars calculated as propagated standard errors of the mean of triplicate measurements from each experiment; ${ }^{*} P<0.05$. (b) Expression of miR-143 and miR-145 in the aorta of apolipoprotein E knockout (ApoE KO) mouse fed on normal (ND) and high-cholesterol diets (HFD), analyzed by qRT-PCR, Sno22 RNA was used as internal control; ${ }^{*} P<0.03$. (c) qRT-PCR of expression of miR-143, -145 and -199 in aortic aneurysm in humans, U6 snRNA was used as internal control; ${ }^{\star} P<0.05$

Homozygous miR-143(145) KO mice were viable at birth and did not display any obvious disorders when kept in normal vivarium conditions, indicating that these two miRs are dispensable for development. We first investigated the timing of $\mathrm{miR}-143 / 145$ gene expression through $\beta$-galactosidase staining. We found that the miR-143/145 gene cassette became activated in aorta and bladder at E13.5 (Figure 3d). Hematoxylin-eosin- and $\beta$-galactosidase (LacZ)-stained histological sections of embryos revealed that the tunica media of the aorta and the tunica muscolaris propria of the bladder and intestine expressed lacZ (Figure 3e; Supplementary
Information; Supplementary Figure S1). In postnatal mice, strong $\beta$-gal staining was evident in the outflow tract of the heart (aorta and pulmonary trunk) and coronaries, and in the main cerebral vessels (Figure $3 f)$. Moreover, lacZ co-localized with smooth muscle $\alpha$-actin (Figure $3 \mathrm{~g}$ ).

Histological examination revealed that the structure of the aorta of homozygous miR-143(145) KO mice was different to the WT (Figure 4a; Supplementary Information; Supplementary Figure S1). Measurement of the absolute media thickness and calculation of the media area/lumen area ratio confirmed this observation. These results suggest that probably there is a defect in the development of the SMC layer in miR-143(145) KO mouse.

Moreover, histological analysis of $\mathrm{KO}$ and WT aortas after acute (TAC) and chronic (angiotensin II (Angll) treatment) pressure stress does not show any major difference between the two groups (data not shown).

VSMCs of the miR-143(145) KO mouse are dedifferentiated. Small formations within the intimal layer of the aorta were often observed in miR-143(145) KO mice. Staining for smooth muscle $\alpha$-actin revealed that these formations were accumulations of VSMCs, indicative of migration of VSMCs from the tunica media to the tunica intima (Figure 4b). Phospho-histone H3 and cleaved Caspase-3 activity evaluated by immunostaining on WT and $\mathrm{KO}$ aortas did not show any difference of proliferation and apoptotic ratio between the two groups (data not shown). Moreover, the colon of miR-143(145) KO mice had a classical megacolon phenotype (Supplementary Information; Supplementary Figure S1), which can be caused by hyperproliferation of SMCs, as evidenced in a mouse model that expresses SV40 large antigen in visceral SMCs. ${ }^{15}$ We tested whether miR-143 and miR-145 altered proliferation in vitro. In an uptake study using A7r5 cells - a secondary rat-VSMC line - we found a $50 \%$ reduction in incorporation of $\left[{ }^{3} \mathrm{H}\right]$ thymidine into A7r5 cells transduced with Ad-miR-143 or Ad-miR-145, compared with a control miRNA-carrying Ad (Figure 4c). We also isolated VSMCs from WT and KO mice, and tested their ability to migrate toward platelet-derived growth factor (PDGF) a known chemoattractant for VSMCs. ${ }^{16} \mathrm{KO}$ cells were more migratory than WT cells (Figure $4 d$ ). Together, these data suggest that the miR-143/145 gene is involved in the control of both proliferation and motility of VSMCs.

Ultrastructural analysis of miR-143(145) KO aorta revealed that VSMCs had a dilated rough endoplasmic reticulum supporting aligned ribosomes, and that these cells contained flocculent material typical of synthetically active VSMCs (Figure 5a). Moreover, transmission electron microscopy analysis of aorta from $\mathrm{miR} \mathrm{KO}$ mice revealed also the presence of extended areas of ECM degradation around the SMCs, which presented elongated structures, indicating the presence of migrating/dedifferentiated SMC (Figure 5b). In addition to these morphological features, a functional analysis of the vasculature of miR-143(145) $\mathrm{KO}$ mice revealed that they had a blunted response to vasopressive stimuli: when miR-143(145) KO mice were treated with a hypertensive dose of Angll, ${ }^{17}$ systolic pressure did not rise as much as WT mice (Figure 6a). These structural and functional observations - migration, hyperproliferation, increase protein 


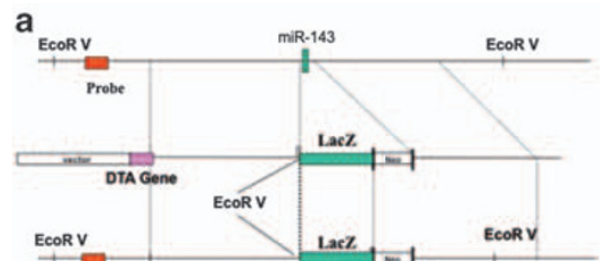

b

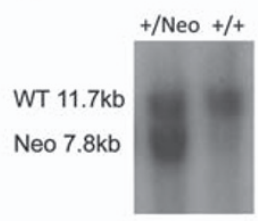

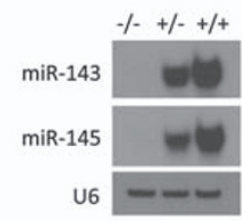

d

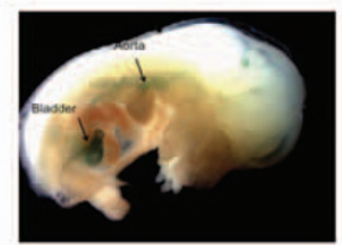

f

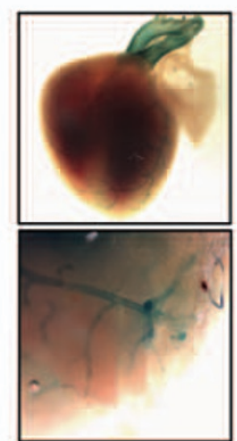

e

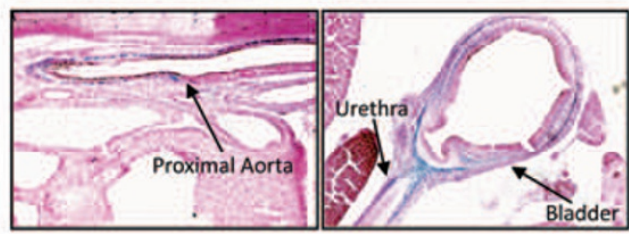

g $\beta$-galactosidase

DAPI g

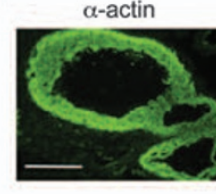

merge

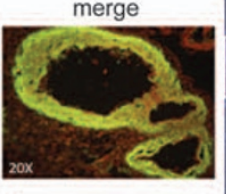

Figure 3 Targeting of miR-143(145). (a) Strategy used to generate miR-143 mutant mice by homologous recombination. (b) Southern blot of genomic DNA from ES cells. (c) Northern blot of miR-143 and miR-145 in aortas of mice wild type $(+/+)$, heterozygous $(+/-)$ and homozygous $(-/-)$ for miR-143/145 deletion. U6 RNA serves as a loading control. (d) Visualization of lacZ-expressing structures in an embryo at E13.5. Arrows indicate $\beta$-galactosidase staining of aorta and bladder. (e) lacZ expression on paraffin sections of embryo at E13.5. (f) lacZ expression on whole heart and brain of P1 mice. (g) Immunofluorescence for smooth muscle $\alpha$-actin and $\beta$-gal on a frozen section of P1 heart. DAPI was used to visualize nuclei. Scale bar $=20 \mu \mathrm{m}$

synthesis and blunted hypertension response - are consistent with the notion that SMCs of miR-143(145) KO mice have a dedifferentiated rather than a differentiated phenotype. In addition, we analyzed mRNA expression profiles of aortas from KO and WT animals using Illumina GeneChips $(n=3$ per genotype) (Illumina, San Diego, CA, USA). Interestingly, we noted a loss of the expression of several markers of differentiated SMCs and an increase in the expression of skeletal muscle markers (Table 1). In support of this, qRT-PCR confirmed the downregulation of two VSMC-differentiation markers, smooth muscle $\alpha$-actin and smooth muscle myosin heavy chain, in the aorta of adult miR-143(145) KO mouse compared with WT (Figure 6b). We hypothesize that miR-143 and miR-145 are necessary for correct maturation of VSMCs during development and might be involved in phenotypic switching in disease states.

Finally, to confirm all these findings in a WT animal model, we performed balloon injury experiment in rat carotids. AdmiR-143 or Ad-miR-145 were administered to WT rats immediately after subjecting them to balloon injury of the carotid artery. We found that balloon-injury-induced neointimal formation was significantly reduced when the carotid artery was locally perfused with adenovirus expressing either miR-143 or miR-145 (Figure 7), demonstrating that miR-143 and -145 also control the proliferation and migration of VSMCs in intact vessels in vivo.

\section{Discussion}

We show here that the miR-143/145 cluster is involved in maintaining the differentiation status of VSMCs, and we propose that their dysregulation underlies the histological changes observed in vessels in pathologies such as atherosclerosis and restenosis.

miR-143 and miR-145 are clustered together on chromosome 5 in humans and chromosome 18 in mice and rats, and originate from the same transcriptional unit. ${ }^{14}$ Thus, the LacZ$\mathrm{Neo}$ insertion in our KO model affects the expression of both miRs. Previous reports have shown miR-143 expression in heart, testes and gut of the zebrafish; ${ }^{18}$ heart, spleen and brain of adult mouse, ${ }^{19}$ and heart, spleen, brain, liver, pituitary, thyroid, pancreas, ovary, testes, prostrate and placenta of adult mice and humans. ${ }^{20}$ At the cellular level, miR-143 is strongly expressed in adipocytes, ${ }^{21}$ osteoclasts ${ }^{22}$ and SMCs of myometrium ${ }^{23}$ and vessels. ${ }^{24}$ We suggest that these genes are needed for correct morphogenesis of the smooth muscle layer of the vascular system and of the gastrointestinal and urogenital tracts, as observed in the mouse from 
a
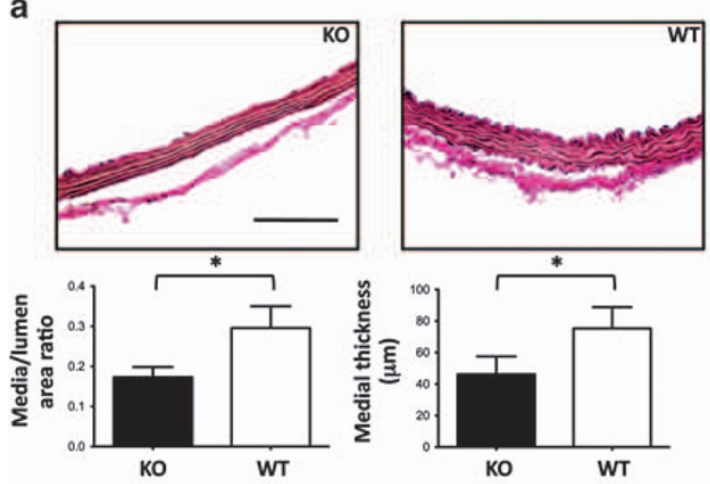

b

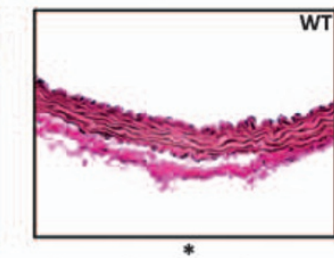

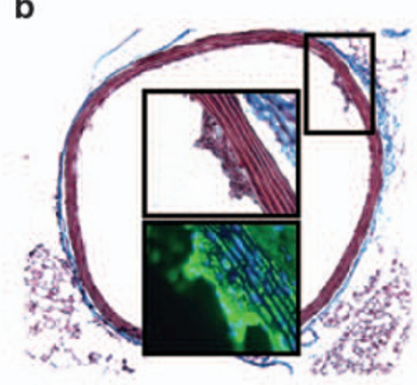

c

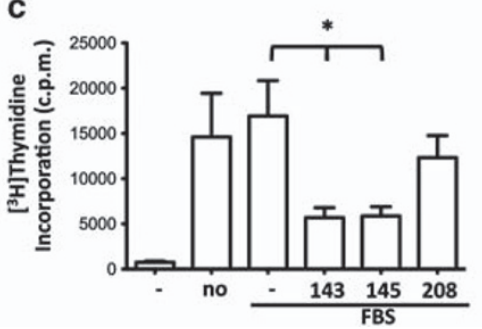

d

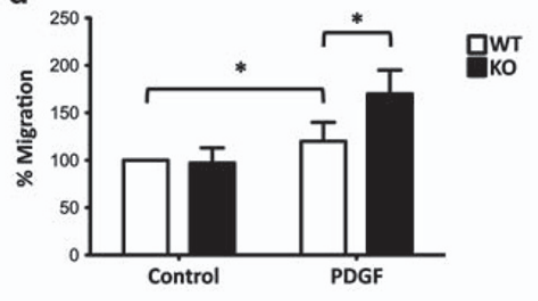

Figure 4 Morphological changes in the vasculature of miR-143(145) KO mice. (a) Van Gieson staining for elastin in aorta from miR-143(145) knockout (KO) and wild-type (WT) mice (top), and analysis of the arterial thickening (bottom) through calculation of the media area/lumen area ratio. Scale bar $=40 \mu \mathrm{m}$. (b) Masson trichrome (whole section and top inset) and smooth muscle $\alpha$-actin immunofluorescence staining (bottom inset) of a cross-section of aorta from an miR-143(145) KO mouse. Original magnification $\times 10$ (whole section), $\times 40$ (insets). (c) Proliferation assay of FBS-treated A7r5 cells. No, no adenovirus; -, Ad-Empty; 143, Ad-miR-143; 145, Ad-miR-145; 208, Ad-miR-208; ${ }^{\star} P<0.05$. (d) Chemotaxis assay of primary VSMCs from WT and KO mice. All measurements were calculated as percent of control (WT) and error bars calculated as propagated standard errors of the mean of triplicate measurements from each experiment; ${ }^{\star} P<0.05$

E13.5 onward. The miR-143/145 genes are upregulated during development and remain constitutively expressed in SMCs of adult organs. Recently, a critical role for miR-143 and -145 in smooth muscle cell fate and plasticity has been reported using a transgenic mouse model in which an enhancer sequence of miR-143 and -145 was driven by the LacZ gene. ${ }^{14}$ In that report, expression of miR-143 and -145 was first detected in cardiac progenitors before expression in VSMCs. These data differ from those reported here, in which we found that expression of the miRs is restricted to SMC, with little expression in the developing heart. These differences may be explained by the different methods used to evaluate expression. As we did not observe gross defects in vessel formation during embryogenesis, it is likely that these miRs are important in the fine tuning of smooth muscle cell physiology but not absolutely required for development.

Analysis of aortas from the miR-143(145) KO mice revealed that loss of these miRs is associated with dedifferentiation. We detected pro-synthetic ultrastructural changes in the ER, decreased expression of differentiation-associated markers and reduction in response to hypertension stimuli. We conclude that the miR-143/145 gene is necessary for maintaining a differentiated SMC phenotype in the adult. Furthermore, we hypothesize that reversibility of this function is important to maintain the plasticity of VSMCs. This conclusion is consistent with the upregulation of miR-143 during differentiation of human pre-adipocytes into adipocytes, and the inhibition of adipocyte-specific gene expression and accumulation of triglycerides when miR-143 is knocked down. ${ }^{21}$ In that report, ERK5, which is known to regulate cell growth and proliferation, was a predicted target gene. We have presented experiments suggesting that miR143 (145) loss promotes both proliferation and chemotaxis of VSMCs. In the future it will be important to dissect the mechanism(s) by which these miRs regulate cell motility.

It is notable that the only apparent in vivo manifestation of the loss of miR-143/145-gene expression in our constitutive $\mathrm{KO}$ model is an aorta with a thinner wall than WT. This may seem at odds with the notion that decreased expression of miR-143 and miR-145 is associated with an increased migratory state of VSMCs in vitro when they are adequately stimulated. The appropriate response of a vessel in vivo requires a concerted regulation network that comprises intracellular mechanisms inherent to VSMCs, and crosstalk between all the cell types that make up the vessel wall. We speculate that the miR-143/145 gene controls only VSMCspecific events; this is supported by the fact that other cell types of the vessel, such as endothelial cells, do not express miR-143 or miR-145 (data not shown). In vivo, therefore, some of the effects of mis-expression of the miR-143/145 gene alone might become masked by counter balancing regulatory signals originating from the other cell types present.

We have shown that increased hemodynamic stress is associated with downregulation of miR-143/145 gene expression in the aorta of WT mice. The pathological states that induce this downregulation are varied and include coarctation and altered vessel histology such as that occurring with atherosclerosis. The relevance of this finding to human vessel 
pathology was substantiated by the fact that the gene was found deregulated in human aortic aneurysms. miR-143 and miR-145 were also shown to be downregulated during

a

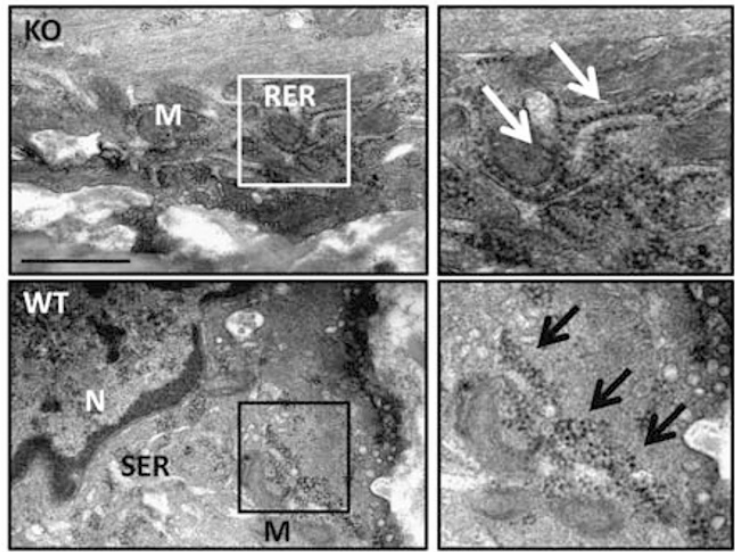

b

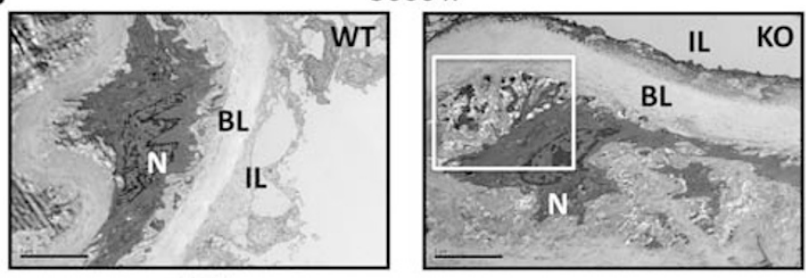

$5500 x$

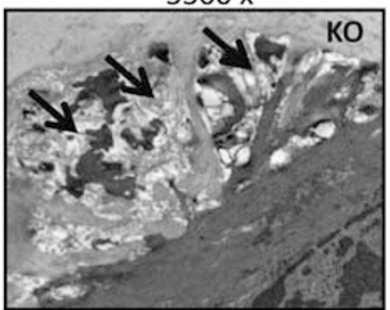

Figure 5 Electron microscopy on mouse aorta. (a) Electron microscopy micrographs of miR-143(145) KO (KO) and wild-type (WT) aorta. M, mitochondria; RER, rough endoplasmic reticulum; SER, smooth endoplasmic reticulum; N, nuclei. Arrows indicate aligned ribosomes present on active RER, whereas they are organized in clusters all around the SER in WT aorta. Scale bar $=1000 \mathrm{~nm}$. (b) Micrograph of WT and miR-143/145 KO aorta (top panel), higher magnification image of the $\mathrm{KO}$ aorta on the bottom panel. N, nucleus; BL, basal lamina; IL, intima layer. Arrows indicate areas of ECM around a KO cell that shows cell protrusions. Scale bar $=5 \mu \mathrm{m}$ neointimal formation in the carotid artery of the rat, ${ }^{24}$ although targets and mechanisms were not reported on. A very recent report described miR-145 regulation of smooth muscle cell differentiation and neointima formation after injury of the rat carotid artery. ${ }^{25}$ We now extend these findings to include miR-143. Our data suggest that miR-143/145-gene expression functions as a brake to dedifferentiation rather than a stimulus for differentiation. We propose, therefore, that the pathogenic role of the mir-143/145 gene is related to its control of phenotypic switching of VSMCs: pathological stimuli induce an inappropriate downregulation of miR-143 and miR-145, and this in turn facilitates dedifferentiation. The consequent increase in migratory ability would then be at the basis of, for example, neointimal formation and progression in atherosclerosis and restenosis, and the focal SMC contractile dysfunction observed in the aortic media and the vasa vasorum with aneurysm.

A growing body of evidence has documented the pathogenic role of the miR-143/145 gene in cancer. Chromosomal deletion at $5 q 32$ - leading to loss of expression of the miR$143 / 145$ gene - was found in colon ${ }^{26}$ and breast ${ }^{27}$ adenocarcinomas. Both miR-143 and miR-145 are also downregulated in most $\mathrm{B}$-cell malignancies, ${ }^{28}$ as well as in cell lines derived from other types of cancers. ${ }^{26}$ Moreover, the miR$143 / 145$ gene becomes downregulated during the transformation of myometrium SMCs into leiomyoma SMCs. ${ }^{23}$ All these findings are consistent with proliferative/migratory status of dedifferentiated VSMC lacking the miR-143 and -145 that we have documented here. Eligible targets, apart from ERK5, included methylenetetrahydrofolate reductase, associated with aberrant DNA methylation; the $\mathrm{Na}$ - and K-dependent ATPase A subunit (ATP1A1); and KRAS (V-Ki-ras2 Kirsten rat sarcoma viral oncogene homolog), a molecular on/off switch that recruits and activates proteins necessary for the propagation of growth-factor signaling. ${ }^{29}$ Thus, miR-143 and miR-145 are suggested to have important anti-oncogenic roles.

We have shown that miR-143 and -145 are important regulators of plasticity of VSMCs, which has pertinence for the pathogenesis of vascular disease. Future investigations using conditional KO mice will be instrumental in identifying other targets of the miR-143/145 gene, to define in more depth the various pathways controlled by miR-143(145) in VSMCs. Furthermore, our current studies are focused on analyzing further the pathogenic roles of miR-143 and -145 in human vascular diseases.
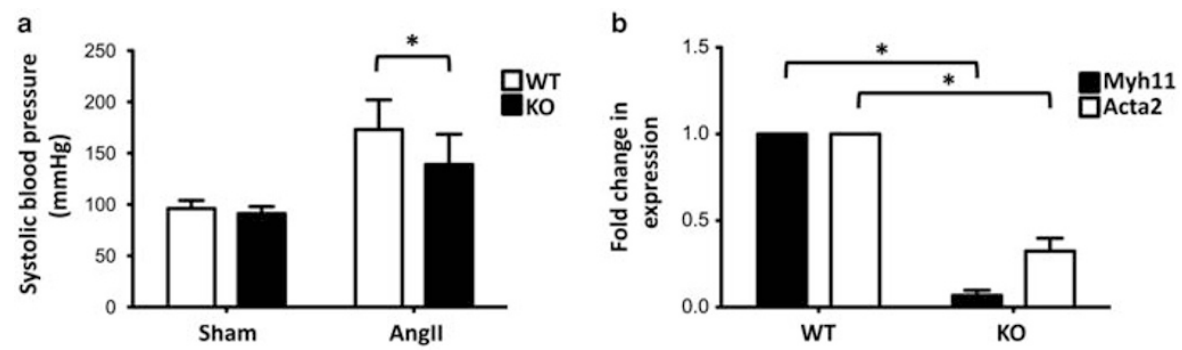

Figure 6 Functional changes in the vasculature of miR-143(145) KO mice. (a) Blood pressure of saline- (Sham) and angiotensin II (Angll)-treated WT and miR-143/145 KO mice after 2 weeks; ${ }^{*} P<0.03$. (b) qRT-PCR on adult aorta from WT and KO mice for smooth muscle myosin heavy chain (Myh11) and smooth muscle $\alpha$-actin (Acta2). GAPDH RNA was used as internal control; data were plotted and analyzed as described in Figure 2; ${ }^{*} P<0.05$ 


\section{Materials and Methods}

Animals and Angll infusion. Animals were housed in accordance with the guidelines of the American Association for Laboratory Animal Care. The miR-143(145) $\mathrm{KO}$ mice were generated using a genomic miR-143 fragment isolated from a 129SVJ library (Stratagene, La Jolla, CA, USA) and used to construct the miR-143 targeting vector using standard techniques. One copy of the LacZ/miR-143 gene was inserted into the targeting vector along with the neo cassette in the place of the genomic miR-143 location. The targeting vector was then linearized with EcoRV and electroporated into R1 embryonic stem (ES) cells. ES clones were screened for homologous recombination by Southern blot analysis. For the Angll experiment, mice were anesthetized by intraperitoneal injection of xylazine $(5 \mathrm{mg} / \mathrm{kg})$ and ketamine $(80 \mathrm{mg} / \mathrm{kg})$

Table 1 Selected transcripts detected upregulated and downregulated in miR143/145 KO versus WT aortas

\begin{tabular}{lll}
\hline Gene title & Gene symbol & FC \\
\hline Troponin C2, fast & Tnnc2 & 8.9 \\
Myosin light chain, phosphorylatable, & Mylpf & 6.4 \\
fast skeletal muscle & & \\
Actin, $\alpha 1$, skeletal muscle & Acta1 & 5.9 \\
Troponin I, skeletal, fast 2 & Tnni2 & 5.5 \\
Actin, $\alpha$ 2, smooth muscle, aorta & Acta2 & 0.6 \\
Myosin, heavy polypeptide 11, smooth muscle & Myh11 & 0.6 \\
Smoothelin & Smtn & 0.5
\end{tabular}

Expression values are ratios of $\mathrm{KO}$ aortas expression values versus WT from adult mice $(n=3 / 3)$. Selected skeletal and smooth muscle differentiation molecules are shown $(P<0.05)$. FC, fold change
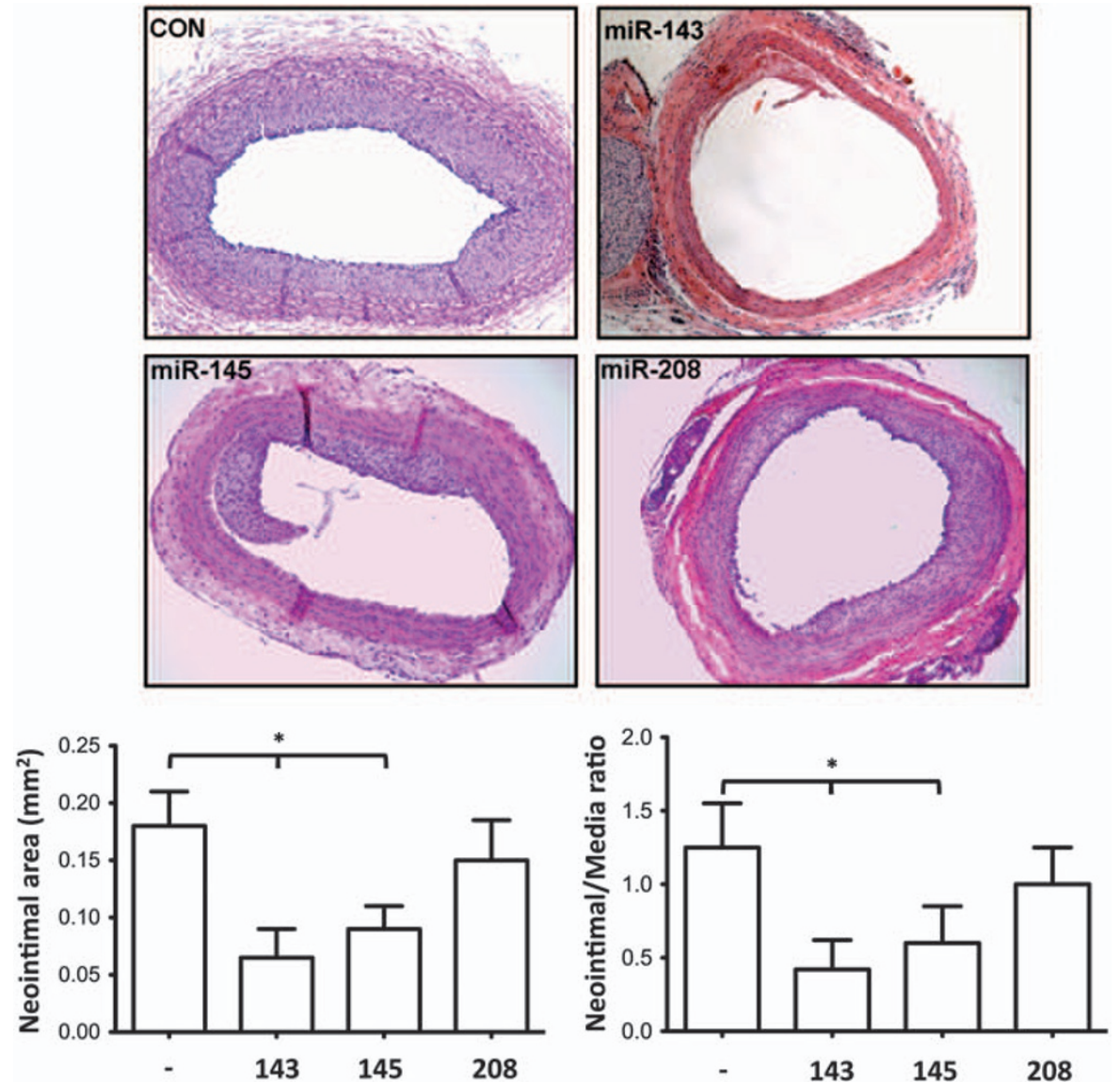

Figure 7 Effects of miR overexpression in rat carotid arteries after balloon injury. Representative cross-sections (14 days after balloon injury in vessels transduced with different miR expressing adenoviral vectors) (top panel). Neointimal area and neointimal/media ratio quantifications (bottom panel); ${ }^{*} P<0.05$ versus contralateral untreated arteries and ALZET minipumps (Model 1002) containing $1.4 \mathrm{mg} / \mathrm{kg}$ per day Ang II (A-9525; Sigma-Aldrich, St. Louis, MO, USA) dissolved in saline or saline alone (sham treatment) were implanted subcutaneously. Blood pressure was measured using a 1.4F Millar catheter 2 weeks after the implant and the data acquired using the software 'Chart' (http://www.adinstruments.com/). Aortas were collected after perfusion with normal saline until the perfusate cleared and fixed in $4 \%$ paraformaldehyde. Eight animals per group were used and the experiment repeated two times.

Balloon injury and in vivo administration of adenovirus. Balloon injury to rat carotid artery, administration of adenovirus and neointimal quantification were performed as described earlier. ${ }^{30}$ Six animals per group were used, carotids were collected at day 14 and the experiment was repeated two times.

Cell lines. A7R5 rat smooth muscle cells were maintained in DMEM (Invitrogen, Carlsbad, CA, USA) supplemented with $1.5 \mathrm{~g} / \mathrm{l}$ glucose, $4 \mathrm{mM}$ L-glutamine, $10 \%$ fetal bovine serum and penicillin/streptomycin at $37^{\circ} \mathrm{C}$ in a $5 \% \mathrm{CO}_{2}$ atmosphere. Primary vascular SMCs were obtained from WT and KO mouse aorta and purity checked as

Materials. The following antibodies were used: SM $\alpha$-actin (Sigma-Aldrich), $\beta$-galactosidase (Roche, Indianapolis, IN, USA), Pecam (BD Bioscience, La Jolla, CA, USA), phospho-histone H3 (Cell Signaling, Danvers, MA, USA), cleaved Caspase-3 (Cell Signaling), Alexa Fluor 594-conjugated phalloidin was from Invitrogen.

Whole and sectioned embryo analysis. $\beta$-gal staining to visualize lac $Z$ expression was performed as described earlier. ${ }^{13}$

Absolute medial thickness and medial area/lumen area ratio. Morphometric measurements were carried out as described earlier. ${ }^{17}$ described earlier. ${ }^{31}$ 
In situ hybridization. In situ hybridization was performed as described earlier. $^{32}$

Immunohistochemistry. van Gieson's stain and Masson's trichrome staining were performed following the manufacturer's protocol for paraffin sections (Sigma-Aldrich)

Immunofluorescence. Immunofluorescence was performed on OCT frozen sections and paraformaldehyde fixed cells. Sections and cells were permeabilized with PBS-Triton $0.1 \%$, then blocked with PBS-Triton $0.1 \%-B S A 5 \%$ for $1 \mathrm{~h}$, washed and incubated $\mathrm{o} / \mathrm{n}$ with the related antibody at $+4{ }^{\circ} \mathrm{C}$. Antibodies conjugated to Alexa Fluor 488 (Molecular Probes, Carlsbad, CA, USA) were used as secondary antibodies, and nuclei were stained with DAPI. Fluorescence microscopy images were obtained with a Zeiss Axioplan2 microscope equipped with a Zeiss Axiocam HRm CCD camera using Axiovision software (Carl Zeiss Inc., Thornwood, NY, USA)

Electron microscopy. Electronic microscopy was performed at the Veterans Medical Research Foundation. Adult aorta from WT and KO mice were dissected and fixed in $2 \%$ glutaraldehyde in PBS for $24 \mathrm{~h}$. The samples were postfixed and stained with $1 \%$ osmium tetroxide in PBS, stained, then dehydrated in a series of graded ethanol and propylene oxide. The tissues were subsequently embedded in Eponate 12, counter stained with uranyl acetate and lead citrate and sectioned at $90 \mathrm{~nm}$.

$\left[{ }^{3} \mathrm{H}\right]$-thymidine uptake assay. Thymidine uptake assay was performed as described earlier. ${ }^{33}$

Migration assays. Boyden transwell chambers (BD Biosciences) were prehydrated $6 \mathrm{~h}$ before cell application with medium containing $0.1 \%$ fetal calf serum (FCS) placed in the insert chambers and with media containing $10 \%$ FCS in the lower chambers. Cells to be tested were detached from cultures dishes in trypsin/EDTA (Invitrogen). This reaction was stopped by a twofold dilution in $1 \mathrm{mg} / \mathrm{m}$ soybean trypsin inhibitor/PBS (final concentration). Cells were sedimented at $1000 \mathrm{~g}$ for $5 \mathrm{~min}$, suspended in $5 \mathrm{ml}$ PBS, resedimented and finally suspended in $1 \mathrm{ml}$ of the appropriate growth media containing $0.1 \%$ FCS; 10000 cells (counted by automation; Beckman) in $500 \mu$ l of media containing $0.1 \%$ FCS were placed into each Boyden chamber. The appropriate growth media containing $0.1 \%$ FCS + PDGF $(10 \mathrm{ng} / \mathrm{ml})(750 \mu \mathrm{l})$ was placed in the lower chamber to facilitate chemotaxis. Invasion assays were run for $6 \mathrm{~h}$. Non-migrating cells were removed from the insert chambers using cotton swabs; then the cells that passed through the membrane were stained with $0.09 \%$ crystal violet $/ 10 \%$ ethanol. After removing excess stain by washing with deionized water, the stain was eluted with $200 \mu$ l of elution buffer (1 part acetate buffer [pH 4.5]: 2 parts ethanol: 1 part deionized water). The optical density of $100 \mu \mathrm{l}$ of the eluted stain was measured at $570 \mathrm{~nm}$ (96-well plate reader, Dynex Technologies, Chantilly, VA, USA). All assays were conducted in triplicate.

Gene expression analysis. Aortas were dissected from PBS-perfused animals, adventitia was removed and total RNA was isolated using the TRlzol method (Invitrogen). The RNA quality, labeling, hybridization and data analysis were performed by the BIOGEM array facility at UCSD, using the mouse MouseWG-6_v2 Illumina platform. Three mice for each genotype were analyzed.

RNA quantification. For qRT-PCR analysis, total RNA was extracted using the TRIzol reagent (Invitrogen) according to the manufacturer's protocol. cDNA was prepared using SuperScript Reverse Transcriptase cDNA kit (Invitrogen). Sybr green qPCR was performed using the following primers: Acta2-5' (5-CTGACAG AGGCACCACTGAA-3), Acta2-3' (5-CATCTCCAGAGTCCAGCACA-3), Mhy11-5' (5-GACAACTCCTCTCGCTTTGG -3), Mhy11-3' (5- GCTCTCCAAAAGCAGGT CAC -3), GAPDH-5' (5-GACGGCCGCATCTTCTTGT-3), GAPDH-3' (5- CACACC GACCTTCACCATTTT-3). For miR qRT-PCR, total RNA was extracted using TRIzol (Invitrogen); primers and probes specific for human miR-143, miR-145 and miR-199 and internal control, Sno202 RNA and U6 snRNA were purchased from Applied Biosystems (Foster City, CA, USA); amplification and detection were performed with 7300 Sequence Detection System (Applied Biosystems), using 40 cycles of denaturation at $95^{\circ} \mathrm{C}(15 \mathrm{~s})$ and annealing/extension at $60^{\circ} \mathrm{C}(60 \mathrm{~s})$. This was preceded by reverse transcription at $42^{\circ} \mathrm{C}$ for $30 \mathrm{~min}$ and denaturation at $85^{\circ} \mathrm{C}$ for $5 \mathrm{~min}$. Northern blotting was performed to confirm the expression levels of miR-143 and miR-145. Probes, anti-sense oligonucleotides against mature miR-143, miR-145 and U6 were locked-nucleic-acid based (Exiqon, Vedbaek, Denmark).
Statistical analysis. RNA, arterial thickening, arterial pressure, proliferation and migration values were compared using two group Student's $t$-test or two-way ANOVA test. A value of $P<0.05$ or less was considered to be statistically significant.

Acknowledgements. This work was supported by EUGeneHeart (LSHM-CT2005-018833), Italian Ministry of Education and Fondation LeDucq grants to GC. The SAC laboratory is supported by the National Cancer Institute and the Mathers Foundation. We are grateful to Dr. Costanza Emanueli, Dr. Jason Johnson, Dr. Brunella Cristofaro and Dr. Saadeh Suleiman, Bristol University (UK) for the donation of the ApoE KO aortas and to Nancy D Dalton and Yusu Gu, University of California San Diego for technical support

1. Gimona M, Herzog M, Vandekerckhove J, Small JV. Smooth muscle specific expression of calponin. FEBS Lett 1990; 274: 159-162.

2. Sobue K, Hayashi K, Nishida W. Expressional regulation of smooth muscle cellspecific genes in association with phenotypic modulation. Mol Cell Biochem 1999; 190: 105-118.

3. Schwartz SM. Smooth muscle migration in atherosclerosis and restenosis. J Clin Invest 1997; 100: S87-S89.

4. Eberhard A, Kahlert S, Goede V, Hemmerlein B, Plate KH, Augustin HG. Heterogeneity of angiogenesis and blood vessel maturation in human tumors: implications for antiangiogenic tumor therapies. Cancer Res 2000; 60: 1388-1393.

5. Owens GK, Kumar MS, Wamhoff BR. Molecular regulation of vascular smooth muscle cell differentiation in development and disease. Physiol Rev 2004; 84: 767-801.

6. Latronico MV, Condorelli G. MicroRNAs and cardiac pathology. Nat Rev Cardiol 2009; 6 : 419-429

7. Urbich C, Kuehbacher A, Dimmeler S. Role of microRNAs in vascular diseases, inflammation, and angiogenesis. Cardiovasc Res 2008; 79: 581-588.

8. Bartel DP. MicroRNAs: genomics, biogenesis, mechanism, and function. Cell 2004; 116 : 281-297.

9. Krek A, Grun D, Poy MN, Wolf R, Rosenberg L, Epstein EJ et al. Combinatorial microRNA target predictions. Nat Genet 2005; 37: 495-500.

10. Owens GK. Regulation of differentiation of vascular smooth muscle cells. Physiol Rev 1995; 75: 487-517.

11. Rockman HA, Wachhorst SP, Mao L, Ross Jr J. ANG II receptor blockade prevents ventricular hypertrophy and ANF gene expression with pressure overload in mice. $A m \mathrm{~J}$ Physiol 1994; 266: H2468-H2475.

12. Breslow JL. Mouse models of atherosclerosis. Science 1996; 272: 685-688.

13. Zhou Q, Chu PH, Huang C, Cheng CF, Martone ME, Knoll G et al. Ablation of Cypher, a PDZ-LIM domain Z-line protein, causes a severe form of congenital myopathy. J Cell Biol 2001; 155: 605-612.

14. Cordes KR, Sheehy NT, White MP, Berry EC, Morton SU, Muth AN et al. miR-145 and miR-143 regulate smooth muscle cell fate and plasticity. Nature 2009; 460: 705-710.

15. Herring BP, Hoggatt AM, Smith AF, Gallagher PJ. Targeted expression of SV40 large T-antigen to visceral smooth muscle induces proliferation of contractile smooth muscle cells and results in megacolon. J Biol Chem 1999; 274: 17725-17732.

16. Raines EW. PDGF and cardiovascular disease. Cytokine Growth Factor Rev 2004; 15 : 237-254.

17. Zhan $\mathrm{Y}$, Brown $\mathrm{C}$, Maynard $\mathrm{E}$, Anshelevich A, Ni W, Ho IC et al. Ets-1 is a critical regulator of Ang II-mediated vascular inflammation and remodeling. J Clin Invest 2005; 115: 2508-2516.

18. Wienholds E, Kloosterman WP, Miska E, Alvarez-Saavedra E, Berezikov E, de Bruijn E et al. MicroRNA expression in zebrafish embryonic development. Science 2005; 309 : 310-311.

19. Lagos-Quintana M, Rauhut R, Yalcin A, Meyer J, Lendeckel W, Tuschl T. Identification of tissue-specific microRNAs from mouse. Curr Biol 2002; 12: 735-739.

20. Landgraf $P$, Rusu $M$, Sheridan $R$, Sewer $A$, lovino $N$, Aravin $A$ et al. A mammalian microRNA expression atlas based on small RNA library sequencing. Cell 2007; 129: $1401-1414$.

21. Esau C, Kang X, Peralta E, Hanson E, Marcusson EG, Ravichandran LV et al. MicroRNA143 regulates adipocyte differentiation. J Biol Chem 2004; 279: 52361-52365.

22. Palmieri A, Pezzetti F, Brunelli G, Martinelli M, Scapoli L, Arlotti M et al. Medpor regulates osteoblast's microRNAs. Biomed Mater Eng 2008; 18: 91-97.

23. Pan $Q$, Luo $X$, Chegini $N$. Differential expression of microRNAs in myometrium and leiomyomas and regulation by ovarian steroids. J Cell Mol Med 2008; 12 : 227-240.

24. Ji R, Cheng Y, Yue J, Yang J, Liu X, Chen $\mathrm{H}$ et al. MicroRNA expression signature and antisense-mediated depletion reveal an essential role of MicroRNA in vascular neointimal lesion formation. Circ Res 2007; 100: 1579-1588. 
25. Cheng $Y$, Liu X, Yang J, Lin $Y, X u$ DZ, Lu $Q$ et al. MicroRNA-145, a novel smooth muscle cell phenotypic marker and modulator, controls vascular neointimal lesion formation. Circ Res 2009; 105: 158-166.

26. Michael MZ, SM OC, van Holst Pellekaan NG, Young GP, James RJ. Reduced accumulation of specific microRNAs in colorectal neoplasia. Mol Cancer Res 2003; 1: 882-891.

27. Iorio MV, Ferracin M, Liu CG, Veronese A, Spizzo R, Sabbioni S et al. MicroRNA gene expression deregulation in human breast cancer. Cancer Res 2005; 65: 7065-7070.

28. Akao $Y$, Nakagawa $Y$, Kitade $Y$, Kinoshita T, Naoe T. Downregulation of microRNAs-143 and -145 in B-cell malignancies. Cancer Sci 2007; 98: 1914-1920.

29. Tong AW, Nemunaitis J. Modulation of miRNA activity in human cancer: a new paradigm for cancer gene therapy? Cancer Gene Ther 2008; 15: 341-355.
30. Indolfi C, Avvedimento EV, Rapacciuolo A, Di Lorenzo E, Esposito G, Stabile E et al. Inhibition of cellular ras prevents smooth muscle cell proliferation after vascular injury in vivo. Nat Med 1995; 1: 541-545.

31. Ray JL, Leach R, Herbert JM, Benson M. Isolation of vascular smooth muscle cells from a single murine aorta. Methods Cell Sci 2001; 23: 185-188.

32. van Rooij E, Sutherland LB, Qi X, Richardson JA, Hill J, Olson EN. Control of stressdependent cardiac growth and gene expression by a microRNA. Science 2007; 316 : 575-579.

33. Goncharova EA, Lim P, Goncharov DA, Eszterhas A, Panettieri Jr RA, Krymskaya VP. Assays for in vitro monitoring of proliferation of human airway smooth muscle (ASM) and human pulmonary arterial vascular smooth muscle (VSM) cells. Nat Protoc 2006; 1: 2905-2908.

Supplementary Information accompanies the paper on Cell Death and Differentiation website (http://www.nature.com/cdd) 